\title{
BACTERIOLOGIC FINDINGS IN OZENA *
}

\author{
HERBERT C. WARD \\ From the Research Laboratory of Parke, Davis and Co., Detroit, Michigan
}

Inquiry into the bacteriology of ozena has engaged the attention of a number of workers during the past 25 years.

In the early eighties of the last century, investigations on the part of Fränkel, Löwenberg, Friedländer, Weichselbaum, Paulsen, and particularly the work of Abel $^{1}$, furnished the interesting suggestion that ozena was due to a bacillus called by Abel "Bacillus mucosus Ozaenae." This organism was recognized by him to be identical with Löwenberg's bacillus, ${ }^{2}$ and closely related to Friedländer's pneumobacillus. Abel found it present in 100 cases of ozena, while in 250 normal controls he failed to discover it once. Paulsen ${ }^{3}$ isolated B. mucosus ozenae in 51 cases; Strazza ${ }^{4}$ in 25, Löwenberg ${ }^{2}$ in 16; Marano ${ }^{5}$ in 10; Thost $^{\mathrm{B}}$ in 12 of 17 ; and Hajek $^{7}$ in 7 of 10.

In contrast with these findings there is a marked absence of Abel's bacillus in infections other than ozena. Abel and Paulsen, reporting their own study of several hundred examinations and taking into account all work published up to that time, estimated that B. mucosus occurred in only $1 \%$ of nasal cases and in about $3 \%$ of oral cases. Netter ${ }^{8}$, in an examination of the sputum of 165 healthy persons, found Abel's bacillus 3 times. Rosenthal ${ }^{2}$ in 14 cases found it in 3 , these being tuberculous. Krause ${ }^{10}$ studying 30 cases of influenza discovered it once. Kowalski likewise obtained it once in 16 cases of influenza. Podbielski ${ }^{11}$ in 50 oral examinations found it once. Besser ${ }^{22}$ investigating the secretions of 81 nasal cases obtained it twice. Wright ${ }^{13}$ reports it totally absent in 10 cases. Paulsen in his study of 27 normal, and 24 acute catarrhal conditions failed to obtain a single culture. When Nicolle and Herbert ${ }^{14}$ conducted their investigation of 1,600 cases of angina, this bacillus was found in pure growth in the mouths of 6 patients.

* Received for publication February 5, 1916.

1 Ztschr. f. Hyg. u. Infektionskrankh., 1896, 31, p. 89. Centralbl. f. Bakteriol., 1893, 31, p. 161 .

Deutsch. med. Wchnschr., 1885, 11, p. 22.

3 Centralbl. f. Bakteriol., 1890, 8, p. 344.

4 Primo Congreso della societa italiana die Laringologia, 1893.

5 Centralb1. f. Bakteriol., 1890, 8, p. 179.

- Deutsch. med. Wchnschr., 1886, 12, p. 161.

7 Berl. klin. Wchnschr., 1888, 25, p. 659.

$s$ Cited by Baumgarten's Jahresbericht, 1890, p. 81.

9 Ein Beitrag zur Kenntniss der Bakterienflora der Mundhohle. Dissertation, 1893.

10 Virchow's Arch. f. path. Anat., 1881, 85, p. 325.

11 Centralbl. f. Bakteriol., 1891, 9, p. 617.

12 Ibid., 1890, 7, p. 151.

13 New York Med. Jour., 1889, 50, p. 92.

14 Ann. de l'Inst. Pasteur, 1897, 11, p. 867. 
Stein $^{15}$ in 51 typical cases of ozena, recognized Abel's bacillus in 44 cases. His 35 healthy controls yielded but 2 cultures. Surveying the entire field he reported the presence of bacterial groups as follows:

Bacillus mucosus-ozenae (in ozena 44 ) $\ldots \ldots \ldots \ldots \ldots \ldots \ldots \ldots, \ldots \ldots \ldots$

Staphylococcus albus and aureus........................

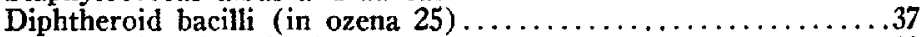

Micrococci, unidentified types....................................

Streptococci and pneumococci........................11

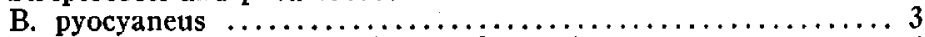

Bacillus mucosus-ozaenae (uncertain type) ................ 4

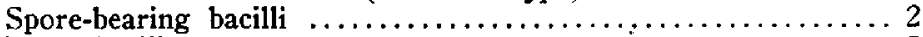

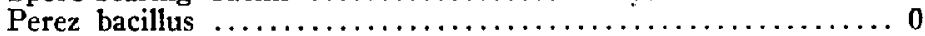

Cobb and $\mathrm{Nagel}^{16}$ in an extensive study of 90 cases of ozena, with special care obtained B. mucosus-ozenae in every examination. Page ${ }^{17}$ isolated Abel's bacillus in 2 cases and supplied fermentation tests for its identification.

Baurowitz ${ }^{18}$ reported Abel's bacillus present in 12 patients with ozena. Seven of these showed pronounced symptoms and the fetor was marked. From 6 he obtained organisms capable of developing the characteristic ozena odor while in culture.

A little later, Perez ${ }^{30}$ reported his bacteriologic findings in 63 cases, 22 of which presented typical symptoms of ozena. The organisms are arranged in the order of their numerical occurrence:

Staphylococcus albus and aureus.......................37

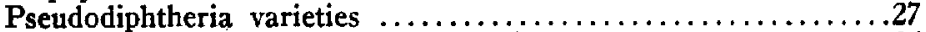

Löwenberg-Abel bacillus (in ozena 17) ..................25

Perez coccobacillus (in ozena 8 ) ...................... 8

Coli-group strains $\ldots \ldots \ldots \ldots \ldots \ldots \ldots \ldots \ldots \ldots \ldots \ldots \ldots, 7$

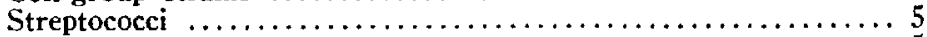

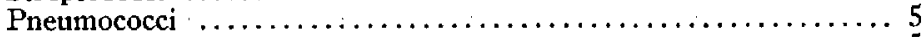

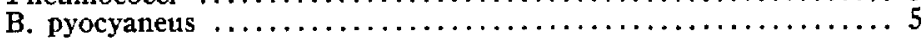

Perez emphasized the finding of a bacterial species which was capable of reproducing the ozena odor while in culture media. He demonstrated that this organism, named by him Coccobacillus foetidus Ozaenae was pathogenic for rabbits, while Abel's bacillus was not. Furthermore, its action was selective, showing affinity for the nasal mucosa, and symptoms developed which simulated those in man. Injected rabbits developed acute and sometimes even chronic conditions, and Perez was able to recover his bacillus from the animal's nasal discharges and to identify it. No such results were to be obtained with Abel's bacillus.

Hofer ${ }^{20}$ in a careful investigation of 14 cases isolated Perez' bacillus in $57 \%$, and Abel's bacillus in $86 \%$. Hofer verified Perez' work with rabbits, concluding with him that his organism was the most important factor in ozena.

Recently a few reports have appeared in this country. Guggenheim ${ }^{2}$ reviewed Hofer's work and noted the finding of Perez' bacillus in a few cases of ozena. His preliminary animal experiments were also in accord with Hofer's. Horn ${ }^{22}$

\footnotetext{
1s Centralbl. f. Bakteriol., 1900, 28, pp. 726, 769.

16 Ann. Oto., Rhinol. and Laryngol., 1912, 21, p. 463.

17 Jour. Med. Research, 1912, 26, p. 489.

18 Centralbl. f. Bakteriol., 1895, 18, p. 719.

1. Ann. de l'Inst. Pasteur, 1899, 13, p. 937.

20 Wien. klin. Wchnschr., 1913, 25, p. 1011.

21 Interstate Med. Jour., 1915, 22, p. 2.

22 Jour. Am. Med. Assn,, 1915, 65, p. 788.
} 
at a recent meeting of the American Medical Association read a paper on the etiology of ozena, in which he favored Perez' bacillus as being the most important etiologic factor.

All these investigations show that the most important bacterial organisms come under two bacteriologic groups; the Friedländer group, represented by Abel's bacillus, and the Bacillus-suipestifer group, represented by Perez' bacillus. In order to extend the present bacteriologic survey in this field, I have studied 50 well-authenticated cases of typical atrophic rhinitis, the majority of which presented ozena conditions.

Pure cultures of the important species were identified biochemically. Special attention was given to the strains obtained from the first few cases. When experience had determined the leading cultural or morphologic characteristics, a less extensive series of tests sufficed for identification purposes. Sero-biologic titrations were employed in the admittance of new strains to the established class, especially in the case of Perez' bacillus. Dr. W. R, Murry and Dr. W. P. Larson, of Minneapolis, by sending me one of Hofer's original cultures, enabled me to establish identification, and so directly correlate our investigations. Pathogenic action was determined with rabbits, these having been reported as promising a differential value.

TYPES OF BACTERIA AND THEIR RELATIVE FREQLENCY IN ATROPHIC RHINITIS

A tabulation of the results shows the relative frequency with which the members of the various bacterial groups appeared. The microscopic picture presents the best record within our reach and is therefore to be preferred, were it not also true that morphologic uniformity prevents identification. The cultural picture is less representative, unimportant species becoming prominent and valuable ones disappearing. It seemed desirable, therefore, to combine the findings obtained by the two methods of study.

The bacteriologic groups are arranged in the order of their frequency in the 50 cases.

Diphtheroids $\ldots \ldots \ldots \ldots \ldots \ldots \ldots \ldots \ldots \ldots \ldots \ldots \ldots \ldots \ldots \ldots \ldots$

Staphylococci, all varieties $\ldots \ldots \ldots \ldots \ldots \ldots \ldots \ldots \ldots \ldots \ldots \ldots \ldots \ldots \ldots \ldots$

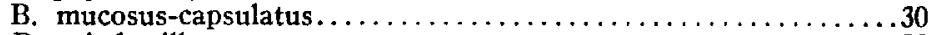

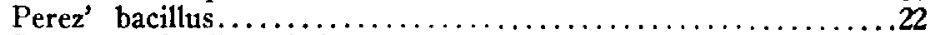

Streptococci, all varieties........................20

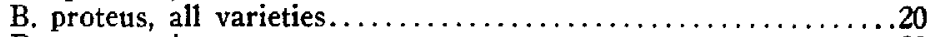

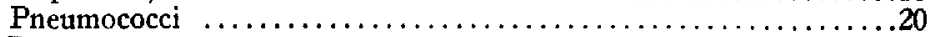

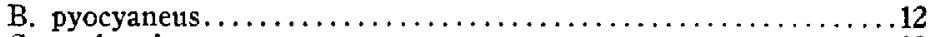

Spore-bearing types................................ 10

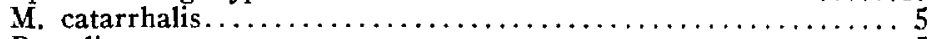

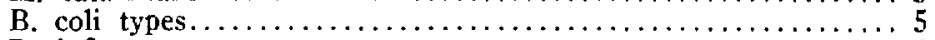

B. influenzae........................... 4

In addition to these predominant forms, there appeared a variety of other organisms whose occurrence was limited, or difficult to verify culturally. 
The diphtheroid group appeared most frequently, a fact in accord with the findings of the earlier workers. The special morphology of this group, together with the use of blood-serum media, explains its predominance.

The micrococci, second in order of frequency, demonstrate that conditions are favorable for their growth and action. Both Perez and Stein have placed this group of bacteria second in their tables.

Abel's bacillus appeared 30 times. The presence of this organism has been considered as typical of bacteriologic conditions in ozena, even by those unwilling to ascribe to it any etiologic significance. The cultures on the agar plates were often pure, while the microscopic picture of the same material revealed a variety of forms. Cobb and Nagel found this bacillus present in $100 \%$ of their cases.

Perez' bacillus, seen first by Horowitz, appeared 22 times in my series. It was identified by culture 15 times.

Perez reports this bacillus as a small, easily stained, gram-negative coccobacillus, showing extreme morphologic variations. It is nonmotile and forms shining transparent colonies. Gelatin is not liquefied or lactose fermented. Milk is not coagulated. Potato shows an abundant yellowish moist growth. It is pathogenic for guinea-pigs, mice, pigeons, and rabbits. All broth cultures give off a characteristic fetid odor, especially in the presence of albuminous media.

Specific agglutinins were easily produced. On the receipt of Hofer's culture, a vaccine was prepared and injected subcutaneously into rabbits. Agglutinins appeared following the 2nd injection, and after 4 doses agglutination of the Hofer strain was positive in a dilution of $1: 3000$.

Perez reported the production in broth of a peculiar odor, developing more or less strongly on incubation, which he used for the identification of his bacillus. With many of our cultures no odor was produced in the original broth. In addition, numerous observations lead us to believe that there exists a small group of bacilli closely allied to Perez', but characterized by motility, and a lack of odor in broth cultures. Also, these strains are unaffected by the Perez agglutinins. Otherwise they appear culturally identical. All of our strains which were admitted to the Perez group, developed this odor, tho it appeared at times to be transient in character. The nature of this decomposition was studied in a few instances. Shurly ${ }^{23}$ believed that the chemical character of the secretions and the histologic elements present determined the character of the decomposition. I consider that this stench is produced by the activity of a specific proteolytic enzyme. To test this property Perez used serum broth; Hofer, broth; Guggenheim, albuminized media. In order to gauge this activity, tests were made for the presence of methyl-mercaptan, employing the isatin

23 Diseases of the Nose and Throat, 1907, p. 376. 
sulfuric acid method, ${ }^{24}$ Flasks of plain broth were inoculated severally with pure cultures of Perez' bacillus, proteus, diphtheroid, Abel's bacillus, and several others. Five cultures of Perez' bacillus produced in broth a strong hydrogen sulfid and mercaptan content; 4 cultures of Abel's organism produced no hydrogen sulfid and no mercaptan; 4 cultures of $B$. proteus produced strong hydrogen sulfid and strong mercaptan; all other tests were negative. These results suggest that the character of the ozena odor is determined by the presence of some of the well-known organic sulfur compounds, especially the mercaptans.

The streptococci appear in the table 5th in order. No special consideration will be given them, since they rarely occurred as the predominating type in the original microscopic pictures.

Sixth in order of isolation stands the proteus group. Just how important this ubiquitous saprophyte may be, it is hazardous to state. Other workers have neglected it entirely. Hajek's organism probably belonged to this group.

It is a luxuriant saprophyte and produces a larger growth and more active enzymic changes in all protein solutions than does Perez' bacillus. It also produces a most nauseating odor in pure culture. This odor is to be distinguished from that produced by other organisms by its suggestive mouselike quality.

\section{PATHOGENIC ACTION OF PEREZ' BACILLUS}

The work reported in the last division of this paper verifies the finding of Perez and Hofer. They proved that this bacillus was pathogenic for laboratory animals; large doses produced death in rabbits, small doses produced marked reaction, seen in the nasal membranes and in the production of excessive mucopurulent secretions, sometimes developing into a chronic condition.

In this study, 28 healthy rabbits received intravenous injections of the Perez culture; 7 rabbits received cultures of B. proteus; 6 of Abel's bacillus, together with 3 cultures of an organism known as Bacillus bronchisepticus, these last serving as controls.

Of the 28 animals injected with Perez' bacillus 12 died-7 after 24 hours, 1 after 3 days, 2 after 3 weeks, and 2 after 4 weeks. All showed a rise of temperature, increased nasal discharge, and in those animals dying last, caking. about the nostrils. Corrosions at the site of inoculation frequently developed, and in the chronic cases emaciation was marked.

The records of the animals recciving B.-proteus injections show that of 7 under test 3 died, at autopsy presenting conditions similar to those observed in the Perez rabbits. After injection all the animals showed pronounced nasal reactions and the organism was recovered from the discharges in 2 cases. Those animals receiving the bronchisepticus cultures developed in 2 instances all the symptoms of snuffles. This condition lasted for several days, recovery following in both instances. In contrast to the foregoing the rabbits which had received the culture of Abel's bacillus showed no reaction and suffered from no infection.

24 Bauer: Ztschr, f. physiol. Chem., 1902, 35, p. 346. 
Future study offers interesting possibilities of reversing the weight of etiologic evidence which is at present ascribed to Perez' bacillus. One of the conclusions arrived at by Herbert and Nicolle in their study of angina was that Abel's bacillus held an important etiologic relationship to certain infections. They stated that it was pathogenic, capable of producing acute and chronic conditions. Allen ${ }^{*}$ has made the repeated observation that the Friedländer bacillus is to be found associated with chronic conditions. Babes, ${ }^{20}$ in his study of rhinoscleroma, suggested the pathogenic relationship of this bacillus to that disease. Abel's bacillus, the original Friedländer's bacillus, and Frosch's bacillus are considered by Perkins ${ }^{27}$ to represent but varieties of one group, under the title of B. mucosus-capsulatus.

If the two organisms are compared on the basis of their pathogenic action in experimental animals, the etiologic importance of the Perez organism seems pronounced. However, when Hofer selected control organisms for his experimental work, he unfortunately chose cultures having practically no value as regards the main point of his work-to prove specific action on the part of Perez' bacillus. His results, together with those of others, would have been much more conclusive had be used organisms found in ozena exudates and infections common to his animals.

It has long been observed that rabbits and other laboratory animals are very susceptible to a form of infection spoken of as "snuffles." Dogs, likewise, are known to succumb to a form of rhinitis presenting symptoms similar to those of ozena in man. In a few reports the idea that the human infection has arisen from the canine is set forth, tho no proof has, as yet, been offered in support of this assumption. Recently, Ferry, ${ }^{28} \mathrm{McGowan},{ }^{29}$ and Torry, ${ }^{30}$ working independently, discovered and proved the relationship of a small bacillus known as B. bronchisepticus to all cases of canine distemper.

B. bronchisepticus is capable of producing in rabbits a type of rhinitis difficult to be distinguished clinically from the pathologic condition produced by Perez' bacillus. In 4 instances rabbits which had received intravenous injections of Perez' bacillus and organisms not Perez' developed "snuffles." From nasal discharges almost pure cultures of B. bronchisepticus were isolated. The close cultural characteristics of these two organisms, especially if differential study is not carried out in detail, might be easily overlooked.

25 The Bacterial Diseases of Respiration, and Vaccines in their Treatment, 1913.

Kolle and Wassermann, Handb. d. pathogen. Mikro-arganismen, 1913, 5, p. 1237.

עT Jour. Infect. Dis., 1904, 1, p. 241.

ss Ibid., 1911, 8, p. 399.

20 Jour. Path. and Bacteriol., 1911, 15, p. 381.

so Jour. Med. Research, 1913, 27, p. 291. 
FREQUENCY OF OZENA TYPES IN OTHER CONDITIONS

In order to measure the comparative values of the Abel, Perez, and proteus organisms in ozena, a control study was made (in co-operation with Dr. W. H. Price, health officer of Detroit, and Dr. D. M. Griswold, in charge of the laboratories) to determine their presence in other conditions. A bacteriologic survey, including 1,400 examinations of cultures from suspected cases of diphtheria, was made. Attention was given to the relative percentages of cases in which the leading types appeared. The results were as follows:

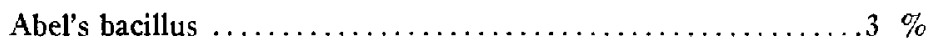

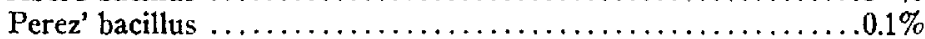

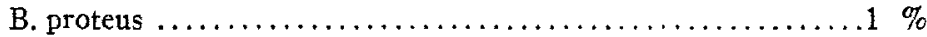

When we contrast with the foregoing the relative percentages of our 50 cases in which these organisms occurred-Abel's bacillus $60 \%$, Perez' bacillus $44 \%$, B. proteus $40 \%$-we find the results agreeing with those obtained by previous observers. Hence the presence of these bacilli in one condition and their absence in other conditions merits serious consideration. It is my opinion that the presence in ozena of Abel's bacillus indicates a pathologic relationship, and I would ascribe to it the role of an etiologic factor. When this bacillus has established a foothold in the nasal mucosa, the way is open for the superimposition of the ozena processes, which are dependent on the entrance of a definite species of bacteria.

\section{CONCLUSIONS}

Since in the ozena stage of atrophic rhinitis Löwenberg-Abel's bacillus is found to predominate and since this organism is seldom present in healthy nasal mucosa, a relationship seems to be indicated between this bacillus and the pathologic condition.

Perez' bacillus can be isolated from an important percentage of cases of ozena. It is not present in normal cases or in infections other than ozena, and rarely exists in atrophic rhinitis.

The peculiar ozena odor is due to the presence of volatile products of protein-decomposition, belonging to the group of the organic sulfids, especially the mercaptans.

Such organic sulfids are produced by both Perez' bacillus and B. proteus, but not by Löwenberg-Abel's bacillus. 
Perez' bacillus produces acute and chronic conditions in animals and it can be obtained from the nasal membranes of such animals.

$B$. bronchisepticus and $B$. proteus are pathogenic for rabbits and capable of producing the same clinical picture with its variations.

In view of the omission of proper controls, further work is necessary to warrant the full acceptance of Perez' bacillus as the most important etiologic factor in ozena. 